\title{
Vortex dynamics in disordered type-II superconductors
}

\author{
J. Müllers and A. Schmid \\ Institut für Theorie der Kondensierten Materie, Universität Karlsruhe, 76128 Karlsruhe, \\ Germany
}

(July 4, 2021)

\begin{abstract}
A field theoretical method is developed which permits us to study the dynamics of vortices in disordered environments. In particular, we obtain a self-consistent system of equations for disorder averaged quantities. Making use of a Hartree-type approximation, we calculate the current-voltage (I-V) characteristics in the flux flow regime. In order to probe incipient melting of the vortex lattice we propose an experiment where interference steps in the I-V characteristics are observed, which arise when a small ac field is superimposed on the constant voltage.
\end{abstract}


Superconductors of type II allow the magnetic field to enter in quantized units of magnetic flux. Commonly one refers to the state with vortex lines present as the Shubnikov phase and in this phase with an applied transport current, an electric field may appear when the vortices are moving.

In an ideal situation, the vortices are ordered in the form of a lattice. Material inhomogeneities (static disorder [1]) tend to prevent the free displacement of the vortices. On the other hand, there are also thermal fluctuations (dynamic disorder) which enhance the vortex motion. Thermal fluctuations are expected to play an important role in high-temperature superconductors [2].

It is appropriate to distinguish two regimes for the current carrying Shubnikov phase depending on whether the vortices are essentially at rest or in motion. The first case is of interest with regard to applications whereas in the second case, intrinsic properties of the vortices and their dynamics will dominate the physics. In order to support the above classification, we wish to draw attention to a recent experiment [3] where by the help of neutron scattering the two regimes can be observed.

The theoretical analysis of these problems was mainly done considering static situations [4] or using perturbation theory for small fluctuations [5]. In the field of research on disordered systems, the techniques used for analyzing problems are the replica method |⿴囗十 and supersymmetry [6]. These remove the so-called denominator problem in the partition function (generating functional) when the average over the disorder is taken. If one has to consider dynamical and interacting systems, the methods referred to above do not seem to work well.

In this context, we recall a real-time method based on the formalism of Martin, Siggia, and Rose (MSR) [7,8] that permits the calculation of averaged dynamical quantities. It was applied by several authors, e.g. in the fields of manifolds in random media [9], polymer melts [10], charge density waves [11], and vortices in type-II superconductors [12].

We present here a reformulation of the MSR theory, which is based on the work of Cornwall, Jackiw, and Tomboulis (CJT) [13,14 and which leads to a self-consistent procedure 
that comprises an expansion not only about the true values of the averaged fields, but also about the true values of the correlation and response functions. The main point of this method is a Legendre transform which yields the velocity as an independent variable instead of the force. We think that this choice is more suitable to describe the situation of an Abrikosov lattice moving in the flux-flow regime. On the other hand, using a rather direct Hartree approximation [9], it was shown that in the static limit ergodicity can be broken and aging effects may occur.

We apply the CJT method to analyze the dissipation due to the motion of vortices in a disordered superconductor. Twenty years ago, this problem was considered by one of us (A.S.) [15] as well as by Larkin and Ovchinnikov [16] in connection with interference steps discovered by Fiory [17,18] in his experiments. With the formalism presented below, the influence both of disorder and of thermal noise on the vortex motion can be calculated in a systematic way. As the high- $T_{c}$ superconductors are built of layers, there is a fundamental interest in the behaviour of two-dimensional systems. In addition, the above mentioned steps are more pronounced if superconducting films are observed. Therefore, as a starting point, we restricted ourselves to the analysis of thin superconducting layers neglecting the vortex tilt.

Let us now consider the classical equation of motion for a two-dimensional system of interacting particles $\{i\}$ (position $\boldsymbol{r}^{i}(t)$, mass $m$, harmonic interaction potential $V_{i j} \boldsymbol{r}^{i} \boldsymbol{r}^{j} / 2$ ) moving dissipatively (viscosity $\eta$ ) in a random potential $U(\boldsymbol{r})$ and driven by a force $\boldsymbol{f}(t)$ as well as by Langevin forces $\boldsymbol{\xi}^{i}(t)$. For the rest of the article we shall use the following conventions: time arguments will be written as index (e.g. $\boldsymbol{r}_{t}^{i}$ ), thermal averages are denoted by $\langle\cdot\rangle_{T}$, and $\langle\cdot\rangle_{U}$ means the average with respect to the random potential, which is taken to be Gaussian. Thus, we have

$$
\begin{aligned}
-\sum_{j} \int d t^{\prime} \mathcal{I}^{R_{t t^{\prime}}^{i j}} \boldsymbol{r}_{t^{\prime}}^{j} & :=m \ddot{\boldsymbol{r}}_{t}^{i}+\eta \dot{\boldsymbol{r}}_{t}^{i}+\sum_{j} V_{i j} \boldsymbol{r}_{t}^{j} \\
& =\boldsymbol{f}_{t}-\nabla U\left(\boldsymbol{r}_{t}^{i}\right)+\boldsymbol{\xi}_{t}^{i},
\end{aligned}
$$

with according mean values and correlations $\langle U(\boldsymbol{r})\rangle_{U}=0$ and $\left\langle U(\boldsymbol{r}) U\left(\boldsymbol{r}^{\prime}\right)\right\rangle_{U}=\Gamma_{U}\left(\boldsymbol{r}-\boldsymbol{r}^{\prime}\right)$ 
for disorder averages and $\left\langle\boldsymbol{\xi}_{t}^{i}\right\rangle_{T}=0$ as well as $\left\langle\boldsymbol{\xi}_{t}^{i} \boldsymbol{\xi}_{t^{\prime}}^{j}\right\rangle_{T}=2 \eta k_{B} T \delta^{i j} \delta_{t t^{\prime}}$ for thermal averages.

The probability for a given path of the particles can be expressed by the functional $\delta$-function, so that the generating functional for the response and correlation functions is given by

$$
\begin{aligned}
Z\left(\left[\boldsymbol{f}_{t}^{i}\right],\left[\boldsymbol{J}_{t}^{i}\right]\right) & =\int \mathcal{D}\left[\boldsymbol{r}_{t}^{i}\right] \mathcal{D}\left[\boldsymbol{R}_{t}^{i}\right] \mathcal{J} \\
& \times e^{i \boldsymbol{R} \bullet_{\bullet}\left[\mathcal{I}^{R} \bullet \boldsymbol{r}+\boldsymbol{f}+\boldsymbol{\xi}-\nabla U(\boldsymbol{r})\right]} e^{i \boldsymbol{r} \bullet \boldsymbol{J}},
\end{aligned}
$$

where e.g. $\boldsymbol{r} \bullet \boldsymbol{q}$ is meant to represent $\sum_{i} \sum_{\alpha} \int d t r_{t}^{\alpha i} q_{t}^{\alpha i}$. Since we have deliberately included the mass $m$ of the particles, the Jacobian $\mathcal{J}=|\delta \boldsymbol{\xi} / \delta \boldsymbol{r}|$ is a constant [19] and may thus be omitted.

The above formulation, which is in essence the MSR theory, allows to perform the disorder and thermal averages immediately. After that, one can write the following compact expression for the generating functional $Z$ :

$$
\begin{aligned}
& Z([\mathbf{q}])=\mathcal{J} \int \mathcal{D}[\mathfrak{r}] e^{i \mathbf{q} \bullet \mathfrak{r}} e^{i S([\mathfrak{r}])}, \\
& S([\mathfrak{r}])=(1 / 2) \mathfrak{r} \bullet \mathfrak{I} \bullet \mathfrak{r}-(i / 2) \boldsymbol{R}_{\mu} \bullet \partial^{\mu} \partial^{\nu} \Gamma_{U}\left(\boldsymbol{r}-\boldsymbol{r}^{\prime}\right) \bullet \boldsymbol{R}_{\nu}^{\prime}
\end{aligned}
$$

where we have introduced the symbols $\mathfrak{r}=(\boldsymbol{R}, \boldsymbol{r}), \mathfrak{q}=(\boldsymbol{f}, \boldsymbol{J})$, and the matrix

$$
\mathfrak{I}=\left(\begin{array}{cc}
2 i \eta k_{B} T & \mathcal{I}^{R} \\
\mathcal{I}^{A} & 0
\end{array}\right) ;
$$

'•' now includes an additional summation over the matrix indices introduced above. Note the structural similiarity between this matrix and the inverse of a propagator in the Keldysh formalism [20].

In order to exploit the field theoretical formalism of Jackiw, Cornwall, and Tomboulis (CJT) [13 we proceed by adding a quadratic source term $(i / 2) \mathfrak{r} \bullet \mathfrak{K} \bullet \mathfrak{r}$ to the exponent in (3). Taking the Legendre transform of the generating functional, that is, of the vacuum diagrams $W([\mathfrak{q}],[\mathfrak{K}])=-i \ln Z$, we arrive at a set of equations which form the starting point of the CJT formalism ( $\overline{\mathfrak{r}}$ means the averaged position and $\mathfrak{G}$ represents the connected correlation and response functions of the particles): 


$$
\begin{aligned}
\frac{\delta W}{\delta \mathfrak{q}} & =\overline{\mathfrak{r}}, \quad \frac{\delta W}{\delta \mathfrak{K}}=\frac{1}{2}(\overline{\mathfrak{r}} \otimes \overline{\mathfrak{r}}+i \mathfrak{G}), \\
\Gamma([\overline{\mathfrak{r}}],[\mathfrak{G}]) & =W([\mathfrak{q}],[\mathfrak{K}])-\mathfrak{q} \bullet \overline{\mathfrak{r}}-\frac{1}{2} \overline{\mathfrak{r}} \bullet \mathfrak{K} \bullet \overline{\mathfrak{r}}-\frac{i}{2} \operatorname{Tr} \mathfrak{G} \bullet \mathfrak{K} ; \\
\frac{\delta \Gamma}{\delta \overline{\mathfrak{r}}} & =-\mathfrak{q}-\mathfrak{K} \bullet \overline{\mathfrak{r}}, \\
\frac{\delta \Gamma}{\delta \mathfrak{G}} & =-\frac{i}{2} \mathfrak{K} .
\end{aligned}
$$

For the effective action $\Gamma$ one can write

$$
\begin{aligned}
\Gamma([\overline{\mathfrak{r}}],[\mathfrak{G}])= & S([\overline{\mathfrak{r}}])-\frac{i}{2} \operatorname{Tr} \ln \mathfrak{I} \mathfrak{G}+\frac{i}{2} \operatorname{Tr} \frac{\delta^{2} S([\overline{\mathfrak{r}}])}{\delta \overline{\mathbf{r}} \delta \overline{\mathbf{x}}^{\prime}} \mathfrak{G} \\
& -\frac{i}{2} \operatorname{Tr} \mathbb{1}+\Gamma_{2}([\overline{\mathbf{r}}],[\mathfrak{G}]) .
\end{aligned}
$$

It has been proven by CJT to all orders, that $\Gamma_{2}$ can be represented as

$$
\Gamma_{2}([\overline{\mathfrak{r}}],[\mathfrak{G}])=-\left.i \ln \int \mathcal{D}[\mathfrak{r}] e^{\frac{i}{2} \mathfrak{\bullet} \bullet \mathfrak{G}^{-1} \bullet \mathfrak{r}+i S_{\text {int }}([\mathfrak{r}],[\overline{\mathfrak{r}})}\right|_{2 P I}
$$

where $S_{\text {int }}$ means an expansion of $S([\mathfrak{r}+\overline{\mathfrak{r}}])$ about $\overline{\mathfrak{r}}$ beginning from the third-order term in $\mathfrak{r}$, and '2PI' indicates that only two-particle irreducible vacuum graphs in a theory with propagators given by $\mathfrak{G}$ and vertices determined by $S_{\text {int }}$ are retained.

Note that eq. (7) implies the Dyson equation

$$
\mathfrak{G}^{-1}=\mathfrak{K}+\tilde{\mathfrak{I}}-2 i \frac{\delta \Gamma_{2}}{\delta \mathfrak{G}}=: \mathfrak{K}+\tilde{\mathfrak{I}}-\tilde{\Sigma}
$$

If we set $\mathfrak{K}=0$ and $\mathfrak{q}=(\boldsymbol{f}, 0)$, which is the physical situation, the above Dyson equation together with the equation of motion (6) constitues an exact self-consistent solution of the problem in consideration.

For further progress we resort to a Hartree-type approximation, which means that in (9) the factor $\exp \left(i S_{\text {int }}\right)$ is replaced by $1+i S_{\text {int }}$. This allows us to perform the path integral exactly because only Gaussian integrals have to be evaluated. In physical situations, $\overline{\boldsymbol{R}}=0$, and $\mathfrak{G}$ has Keldysh structure, i.e.

$$
\mathfrak{G}=\left(\begin{array}{cc}
0 & \mathcal{G}^{A} \\
\mathcal{G}^{R} & \mathcal{G}^{K}
\end{array}\right)
$$


For a demonstration we apply the theory presented above to the simple system consisting of only one particle moving in two dimensions subject to a random potential ("'tilted rough surface"') and to Langevin forces. Specifically, the applied force $\boldsymbol{f}_{t}$ is taken to be constant in time; as a consequence, the mean particle position is $\overline{\boldsymbol{r}}=\boldsymbol{v} t$. We may add that a constant mean velocity requires a sufficiently large driving force, so that the probability for having the particle trapped by a potential well is neglegible. In order to complete the presentation of the simple model, we assume that the random-potential correlator (in Fourier space) is of the form $\Gamma_{U}(\boldsymbol{k})=\Gamma_{U}(0) \exp \left(-k^{2} \xi^{2}\right)$.

Thus, in the overdamped limit the physical scales are determined by the disorder strength $\Gamma_{U}(0)$, the viscosity $\eta$, as well as by the correlation length $\xi$. The self-consistent system of equation in this case reads

$$
\begin{aligned}
\mathcal{G}_{\omega}^{R}= & \frac{\hat{\boldsymbol{v}} \otimes \hat{\boldsymbol{v}}}{(m \omega+i \eta)(\omega+i 0)-\Sigma_{L \omega}^{R}}+ \\
& +\frac{\mathbb{1}-\hat{\boldsymbol{v}} \otimes \hat{\boldsymbol{v}}}{(m \omega+i \eta)(\omega+i 0)-\Sigma_{T \omega}^{R}}, \\
\mathcal{G}_{\omega}^{K}= & \mathcal{G}_{\omega}^{R} \mathcal{G}_{\omega}^{A}\left(\Sigma_{\omega}^{K}-2 i \eta k_{B} T \mathbb{1}\right) \\
\mathcal{S}_{t}^{R}= & \int \frac{d^{2} k}{4 \pi^{2}} \Gamma_{U}(\boldsymbol{k}) \boldsymbol{k} \otimes \boldsymbol{k} e^{i \boldsymbol{k} \boldsymbol{v} t} e^{-i \boldsymbol{k}\left(\mathcal{G}_{0}^{K}-\mathcal{G}_{t}^{K}\right) \boldsymbol{k}} \boldsymbol{k} \mathcal{G}_{t}^{R} \boldsymbol{k}, \\
\Sigma_{\omega}^{R}= & \mathcal{S}_{\omega}^{R}-\mathcal{S}_{0}^{R} \\
\Sigma_{t}^{K}= & -i \int \frac{d^{2} k}{4 \pi^{2}} \Gamma_{U}(\boldsymbol{k}) \boldsymbol{k} \otimes \boldsymbol{k} e^{i \boldsymbol{k} \boldsymbol{v} t} e^{-i \boldsymbol{k}\left(\mathcal{G}_{0}^{K}-\mathcal{G}_{t}^{K}\right) \boldsymbol{k}}, \\
\boldsymbol{f}_{P}= & i \int \frac{d^{2} k}{4 \pi^{2}} \Gamma_{U}(\boldsymbol{k}) \boldsymbol{k} \int d t e^{i \boldsymbol{k v} t} e^{-i \boldsymbol{k}\left(\mathcal{G}_{0}^{K}-\mathcal{G}_{t}^{K}\right) \boldsymbol{k}} \boldsymbol{k} \mathcal{G}_{t}^{R} \boldsymbol{k} .
\end{aligned}
$$

The last line represents the pinning force defined as $\boldsymbol{f}_{P}:=\boldsymbol{f}-\eta \boldsymbol{v}$. Note that we have $\boldsymbol{f} \| \boldsymbol{v}$ by symmetry. The indices ' $L$ ' and ' $T$ ' denote the longitudinal and transverse parts of the self-energy $\Sigma$, and we have used $(\boldsymbol{a} \otimes \boldsymbol{b})^{\mu \nu}:=a^{\mu} b^{\nu}$.

We have solved these equations numerically by iteration, i.e. starting by rather arbitrary given self-energies and performing then repeatedly the above steps. For the velocity $\eta v \gtrsim$ $\Gamma_{U}(0)^{1 / 2} / \xi^{2}$, the iteration converges quite rapidly and yields the force-velocity relation shown in fig. 11. We have found that a diagonal approximation of all matrices, e.g. $\mathcal{G}^{R} \equiv G^{R} \mathbb{1}$, leads to nearly the same curves. 
To check the quality of the Hartee-type approximation, we have examined the energy conservation. If we multiply the equation of motion (11) by the particle velocity, average over random potential and thermal forces, and subtract the unconnected part, we find

$$
\begin{aligned}
\boldsymbol{v} \boldsymbol{f}_{P} & =\eta\left\langle\left\langle[\dot{\boldsymbol{r}}-\boldsymbol{v}]^{2}\right\rangle\right\rangle-\langle\langle\dot{\boldsymbol{r}} \boldsymbol{\xi}\rangle\rangle \\
& \equiv-i \eta \ddot{G}_{t=0}^{K \alpha \alpha}+2 \eta k_{B} T \dot{G}_{t=0}^{R \alpha \alpha} .
\end{aligned}
$$

The last line explicitly yields the relation to the correlation and response functions. Therefore, we can test our approximation by inserting the corresponding functions calculated from eqs. (12) into the conservation relation. The agreement was found to be extremely well, and we believe that the relation holds analytically even after the Hartree-type approximation.

For large velocities, $f_{P}$ and the self-energy $\tilde{\Sigma}$ vanish $\sim 1 / v$. In this limit, a perturbation theory [15] is permissible. On the other hand, for smaller velocities $\eta v \lesssim \Gamma_{U}(0)^{1 / 2} / \xi^{2}$ the fluctuations grow and finally become larger than the mean velocity. In this regime, the importance of the self-energy $\tilde{\Sigma}$ comes into play.

After this preparatory discussion of a viscous particle moving on a rough surface, we will now study the dynamics of vortex lattices. The equation of motion (11) for this system can be cast in the form

$$
m \ddot{\boldsymbol{r}}_{l t}+\eta \dot{\boldsymbol{r}}_{l t}+\sum_{\boldsymbol{l}^{\prime} \neq \boldsymbol{l}} \boldsymbol{D}_{\boldsymbol{l} \boldsymbol{l}^{\prime}} \boldsymbol{r}_{\boldsymbol{l}^{\prime} t}=\boldsymbol{f}_{t}-\nabla U\left(\boldsymbol{l}+\boldsymbol{r}_{l t}\right)+\boldsymbol{\xi}_{\boldsymbol{l} t},
$$

where we have assumed harmonic vortex interactions [21], and $\boldsymbol{l}$ denotes the equilibrium lattice sites. The dissipation is supposed to originate from a Bardeen-Stephen mechanism [22]. In terms of electrodynamical quantities the viscosity is related to the conductivity by $\eta=B \phi_{0} \sigma_{f}$ where $\phi_{0}=\pi \hbar / e$ is the flux quantum. The velocity and force are related to voltage and current by $\boldsymbol{E}=\boldsymbol{B} \times \boldsymbol{v}$ and $\boldsymbol{f}_{L}=\boldsymbol{j}_{T} \times \boldsymbol{\phi}_{0}$ (Lorentz force). The mass of the vortices is small and therefore, it may be omitted at the last step of the calculations.

We shall analyze the flux-flow regime where for constant Lorentz force $f$ the mean vortex positions are supposed to be $\boldsymbol{l}+\boldsymbol{v} t$. One can go through the above sketched steps. In particular, we have done numerical calculations for small lattices and have been able to 
confirm the energy conservation. The expression used for the mean vortex position implies that the vortices experience only small deviations from the equilibrium lattice positions. This assumption should be reasonable for sufficiently large velocities and is confirmed experimentally in a recent letter [3].

Of special interest is a situation where in addition to the dc electric field one has a small ac voltage of frequency $\Omega[17,18]$, so that we have $\langle\langle\dot{\boldsymbol{r}}\rangle\rangle=\boldsymbol{v}+\boldsymbol{w} \Omega \cos \Omega t$. In that case, one can find steps in the $I_{d c}-V_{d c}$ curves at fields $E_{n n^{\prime}}=\left(n^{\prime} / n\right)(\Omega / 2 \pi) \sqrt{2 \phi_{0} B / \sqrt{3}}$. This effect is based on the interference of the applied oscillations with the motion of the periodic vortex lattice. It is completely analogous to the mechanism responsible for the occurence of Shapiro steps [23] in the I-V characteristics of Josephson junctions. There, a mixing of modes occurs due to the nonlinear (periodic) relation of phase and current. For large junctions one finds interference steps for $n^{\prime} \hbar \Omega=n 2 e V_{d c}$. This relation also has been pointed out by Martinoli [24], who considered periodic pinning potentials.

In the case of disorder, the mixing of modes originates from the nonlinearity of the random pinning potential. Thus, one can gain information about the physics of the vortex lattice. From width and height of the interference steps one can infer the shear modulus $c_{66}$ and the correlation $\Gamma_{U}(\boldsymbol{r})$ of the pinning potential. This was done by Fiory [17] using the perturbation theory of Schmid and Hauger [15]. With our method, we may derive improved expressions that are applicable at lower velocities.

For $4 \pi \xi c_{66} \gg \eta v \gtrsim\left(\phi_{0} / 4 \pi B\right)^{1 / 2} \Gamma_{U}(0)^{1 / 2} / \xi^{2}$ one has distinguishable steps and may approximate the self-consistent scheme by the first nontrivial iteration. In addition, we neglect thermal fluctuations and assume the ac amplitude to be sufficiently small, so that one may restrict oneself to the consideration of the steps $E_{n 1}$ [15]; in an approximation, we take the matrix $\tilde{\Sigma}$ from the dc case.

In order to have measurable effects of first order in $w$, one observes the in-phase resistivity $\rho(E)$ [17]. Then, the height of the steps is given by

$$
h_{n}=\frac{3 n^{2} g_{1}^{4} \rho_{f}^{2}}{32 c_{66} \Omega B \phi_{0}} \sum_{m} \Gamma_{U}\left(g_{1} w_{m n}^{1 / 2}\right) w_{m n}
$$


with $w_{m n}=m^{2}+m n+n^{2}$ and $g_{1}=2 \pi\left(2 B / \sqrt{3} \phi_{0}\right)^{1 / 2}$. If we expand $\rho(E)$ linearly near a step voltage where $E \approx E_{n 1}+\delta E_{n} / 2$, we may define the halfwidth of the steps by $\delta E_{n}=$ $h_{n} /\left(2 \rho^{\prime}\left(E_{n 1}\right)\right)$. Thus,

$$
\begin{aligned}
\left(\delta E_{n}\right)^{-1}= & \frac{32 \pi c_{66} \rho_{f} a_{n} n}{E_{n 1}^{2} \phi_{0} g_{1}} \frac{\sum_{m} \Gamma_{U}\left(g_{1} w_{m n}^{1 / 2}\right) w_{m n}}{\sum_{m^{\prime}} \Gamma_{U}\left(g_{1} w_{m^{\prime} n}^{1 / 2}\right) w_{m^{\prime} n}} \times \\
& \times\left\{1-\sqrt{\pi} x_{m n} \exp \left(x_{m n}^{2}\right) \operatorname{erfc}\left(x_{m n}\right)\right\} \\
a_{n}:= & +\frac{2 \pi g_{1}^{2} \rho_{f}^{2}}{\sqrt{3} \phi_{0}^{2} E_{n 1}^{2}} \sum_{m^{\prime}} \Gamma_{U}\left(g_{1} m^{\prime}\right) m^{\prime 4} \\
x_{m n}:= & \frac{\pi^{2} g_{1}^{2} w_{m n} \rho_{f}^{2} \sqrt{a_{n}}}{\sqrt{3 \pi} \phi_{0}^{2} E_{n 1}^{2}} \sum_{m^{\prime}} \Gamma_{U}\left(g_{1} m^{\prime}\right) m^{\prime 2}
\end{aligned}
$$

This result differs from the earlier one [15] by fluctuation effects which appear here in a systematic way. For instance, we obtain $i\left(G_{00}^{K}-G_{\boldsymbol{l}-\boldsymbol{l}^{\prime}, t-t^{\prime}}^{K}\right)=\left\langle\left\langle\left[\boldsymbol{r}_{\boldsymbol{l} t}-\boldsymbol{r}_{\boldsymbol{l}^{\prime} t^{\prime}}-\left\langle\left\langle\boldsymbol{r}_{\boldsymbol{l} t}-\boldsymbol{r}_{\boldsymbol{l}^{\prime} t^{\prime}}\right\rangle\right\rangle\right]^{2}\right\rangle\right\rangle \propto$ $\sqrt{t-t^{\prime}}$ for large $\left|t-t^{\prime}\right|$ and $\propto\left(t-t^{\prime}\right)^{2}$ for small time differences, whereas in 15 a linear time dependence has been considered. In addition, the contribution of $\Sigma^{R}$ is not included in the analysis of [15].

Note that the structure function is given by

$$
S(\boldsymbol{k}, \omega)=\sum_{\boldsymbol{l}} \int d t e^{-i \omega t+i \boldsymbol{k} \boldsymbol{l}+i \boldsymbol{k} \boldsymbol{v} t} e^{-i k^{2}\left(G_{00}^{K}-G_{l t}^{K}\right)}
$$

consequently, the broadening of the interference steps, which is governed by the vortex fluctuations, is directly related to the structure factor. This can be seen e.g. from eqs. (12). Therefore, the width of the steps can give indications for the beginning of a melting transition [25,26] in the vortex lattice.

In summary, we have presented a method that permits to study the dynamics of systems subjected to random potentials and forces. We have combined the formalisms of MSR and CJT and derived a self-consistent system of equations for the averaged particle positions and the correlation and response functions. The advantage of this combination lies in the fact that it is not only an expansion about the mean positions, as it would be the case for a mean field theory, but also one about the full correlation and response functions. Eventually, we have solved the self-consistent system of equations in a Hartree type of aproximation. 
New results have been obtained for a single particle moving on a rough surface in a viscous medium and also, for a lattice of vortices.

We gratefully acknowledge the helpful assistance of U. Eckern and H. Glöckler. This work was supported by the Deutsche Forschungsgemeinschaft. 


\section{REFERENCES}

[1] A.I. Larkin and Yu.N. Ovchinnikov, J. Low Temp. Phys. 34, 409 (1979)

[2] G. Blatter et al., Rev. Mod. Phys. 66, 1125 (1994)

[3] U. Yaron et al., Phys. Rev. Lett. 72, 2748 (1994)

[4] J.P. Bouchaud, M. Mézard, and J.S. Yedidia, Phys. Rev. Lett. 67, 3840 (1991)

[5] M.V. Feigel'man et al., Phys. Rev. Lett. 63, 2303 (1989)

[6] K.B. Efetov, Adv. Phys. 32, 53 (1983)

[7] P.C. Martin, E.D. Siggia, and H.A. Rose, Phys. Rev. A 8, 423 (1973)

[8] R. Phythian, J. Phys. A 10, 777 (1977)

[9] H. Kinzelbach and H. Horner, J. Phys. I (France) 3, 1329 \& 1901 (1993)

[10] T.A. Vilgis, J. Phys. I (France) 1, 1389 (1991)

[11] O. Narayan and D.S. Fisher, Phys. Rev. Lett. 68, 3615 (1992)

[12] L. Radzihovsky and E. Frey, Phys. Rev. B 48, 10357 (1993)

[13] J.M. Cornwall, R. Jackiw, and E. Tomboulis, Phys. Rev. D 10, 2428 (1974)

[14] H.J. He et al., Z. Phys. C 45, 427 (1990)

[15] A. Schmid and W. Hauger, J. Low Temp. Phys. 11, 667 (1973)

[16] A.I. Larkin and Yu.N. Ovchinnikov, Sov. Phys. JETP 38, 854 (1974)

[17] A.T. Fiory, Phys. Rev. B 7, 1881 (1973)

[18] A recent experiment has been published by J.M. Harris et al., Phys. Rev. Lett. 74, 3684 (1995)

[19] A. Schmid, J. Low Temp. Phys. 49, 609 (1982) 
[20] L.V. Keldysh, Sov. Phys. JETP 20, 1018 (1965)

[21] E.H. Brandt, Int. J. Mod. Phys. B 5, 751 (1991)

[22] J. Bardeen and M.J. Stephen, Phys. Rev. 140, A1197 (1965)

[23] S. Shapiro, Rev. Mod. Phys. 36, 223 (1964)

[24] P. Martinoli, Phys. Rev. B 17, 1175 (1978)

[25] D.R. Nelson, Phys. Rev. Lett. 60, 1973 (1988)

[26] R.G. Beck et al., Phys. Rev. Lett. 68, 1594 (1992) 


\section{FIGURES}

FIG. 1. Pinning force $f_{P}(v ; T)$ for a single particle (dimensionless units, i.e. $\left.\eta=1, \xi=1, \Gamma_{U}(0)=1\right)$ 


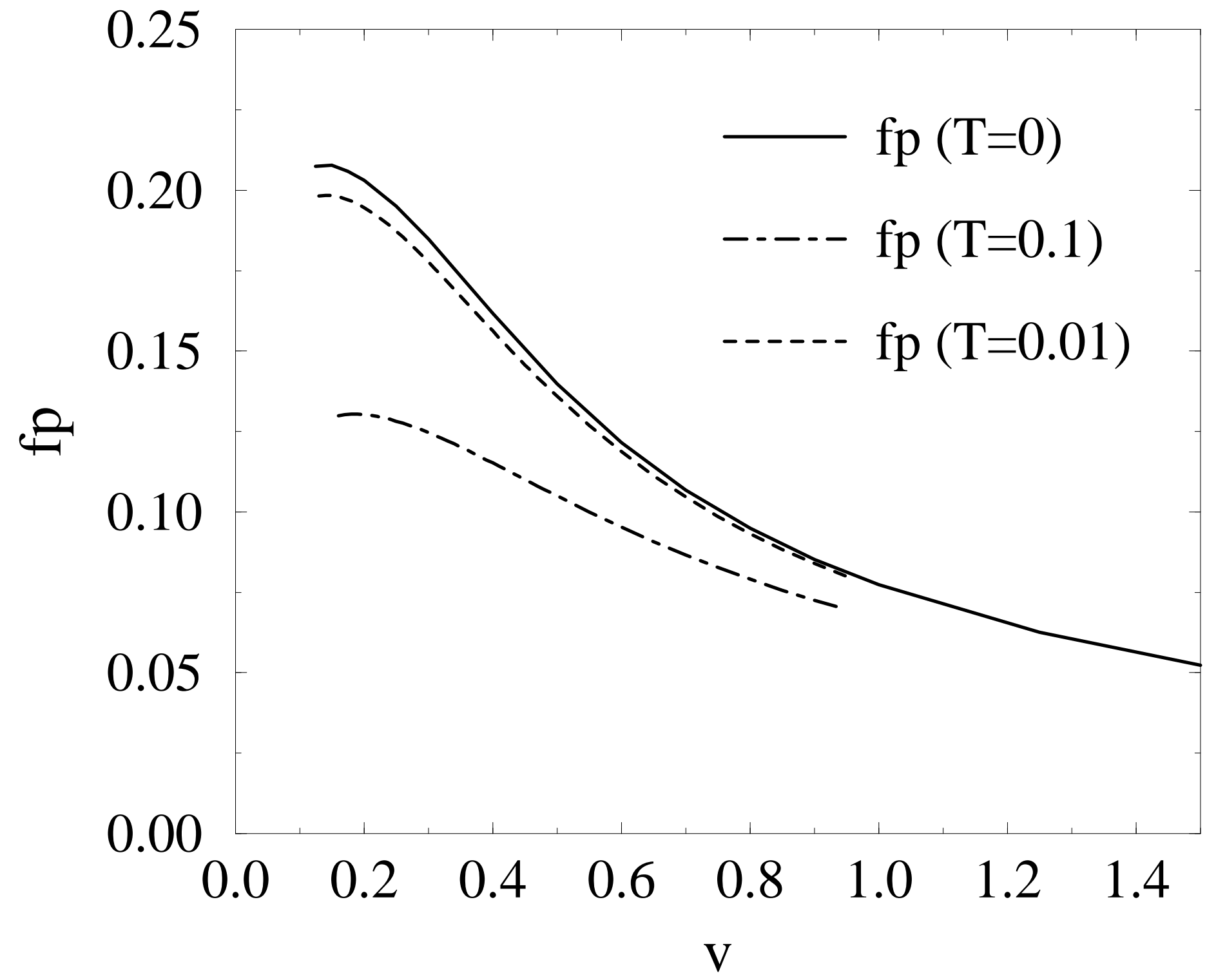

